\title{
Variation in virulence of Septoria tritici Rob ex Desm isolates on wheat
}

\author{
AE Perello ${ }^{1 *}$, CA Cordo ${ }^{1}$, HO Arriaga 2, HE Alippi 3 \\ 1 Comissión de Investigaciones Científicas (CIC) de la Provincia de Buenos Aires; \\ 2 Universidad Nacional de la Plata, Facultad de Agronomía, Cátedra de Cerealicultura; \\ 3 Universidad Nacional de la Plata, Facultad de Agronomía, Cátedra de Fitopatología, \\ 60 y 118 (1900) La Plata, Buenos Aires, Argentina
}

(Received 7 June 1990; accepted 3 May 1991)

\begin{abstract}
Summary - To identify physiologic races, the variation in virulence of 10 local Septoria tritici Rob ex Desm isolates was evaluated on 10 wheat differentials selected in Israel and 7 previously tested Argentine cultivars (cvs). Pycnidial leaf coverage percentage (PC) and necrotic lesion percentage (NL) were used to determine cvs reaction in order to compare their efficiency in isolate differentiation. Isolates behaved differently on the different wheat cvs. The 2 variabies showed which isolates behaved similarly, and which behaved differently; PC was more sensitive in detecting these differences. The ranking of varieties for PC and NL was different for every isolate $\times \mathrm{cv}$ combination, and few ordering patterns coincided (Bobwhite "S", lassul 20 and Bordenave Puán). In general, local isolates induced more PC than NL on national cvs. Two isolates $\left(I_{11}, I_{19}\right)$ behaved in the most virulent manner; 2 others $\left(I_{21}, I_{25}\right)$ provoked the highest resistant reaction percentage on the cvs tested. Isolate $\times \mathrm{cv}$ interaction varied according to the parameter evaluated. The analysis of variance for $\mathrm{PC}$ indicated significant interaction between the 2 main factors $(P=0.01)$ which may be considered as an index of pathogen physiologic specialization. However, a non-significant interaction ( $P=$ $0.01)$ for NL indicated only differences between isolates degree of aggressiveness. Low degree of specificity of the interactions and similar order in the ranking of disease severity on some cvs showed no "true physiologic races". The international set did not supply any major variation range in lesion type and percentage or more consistent cv reactions in differentiating biotypes. Thus, the standardization of research methodologies is necessary to study $S$ tritici-Triticum $\mathrm{sp}$ interactions. This would contribute to the reproducibility of results obtained by different authors throughout the world and thus avoid different interpretations of varietal reaction.
\end{abstract}

\section{virulence / isolates / Septoria tritici / wheat / physiologic races}

Résumé - Variation de la virulence d'isolats de Septoria tritici Rob ex Desm sur blé. La variation de virulence de 10 isolats de Septoria tritici a été évaluée sur 17 variétés de blé sélectionnées : 10 en Israël et en Argentine, pour identifier des races physiologiques (tableau l).

La réaction des variétés est déterminée afin d'estimer l'effet de la méthode d'évaluation de la différenciation des souches et montrer les différences de comportement des variétés de blé par l'emploi de 2 variables:

- le pourcentage de lésions nécrotiques avec pycnides (NL),

- le pourcentage de couverture de la surface foliaire totale par des pycnides (PC).

Les 2 variables, $P C$ et $P N$, permettent de séparer les isolats en fonction de leur comportement, mais avec un net avantage pour PC. La magnitude de la surface nécrosée et la couverture pycnidiale donnent des résultats différents pour chaque combinaison : souche/variété (tableau III). II existe peu de concordance entre les classements (variétés Bobwhite "S", lassul 20 et Bordenave Puán). Les isolats locaux ont, en général, révélé plus de PC que de NL dans les variétés nationales (tableaux $I V$ et $V$ ). Deux d'entre eux $\left(I_{11}\right.$ et $\left.I_{19}\right)$ se sont montrés plus virulents alors que 2 autres $\left(I_{21}\right.$ et $\left.I_{25}\right)$ ont le pourcentage le plus élevé de réactions de résistance sur les 17 variétés testées.

L'interaction souche/variété varie selon le paramètre d'évaluation considéré : une valeur significative $(P=0,01)$ pour $P C$ peut être considérée comme un indice de spécialisation physiologique de l'agent pathogène mais, une interaction non significative $(\mathrm{P}=0,01)$ pour NL n'a montré que des différences dans le degré d'agressivité des isolats. En outre, le faible degré de spécificité des interactions, et l'ordre similaire de l'évaluation de la gravité de la maladie sur cer-

\footnotetext{
* Correspondence and reprints
} 
taines variétés montrent qu'ils n'ont pas mis en évidence de "vraies races physiologiques". L'emploi de la gamme internationale d'hôtes différentiels n'a pas fourni plus de variation pour le type et le pourcentage de lésion, ni de réactions des variétés, plus solides et plus claires, dans le but de différencier des biotypes.

Enfin, il faut insister sur le besoin de standardiser les conditions d'inoculation et d'évaluation des études d'interactions $\mathrm{S}$ tritici/Triticum $\mathrm{sp}$, afin d'assurer la reproductibilité des résultats au niveau mondial et d'éviter les différences d'interprétation de réaction des variétés qui surgissent par l'emploi de méthodologies variables.

virulence / isolats / Septoria tritici / blé

\section{INTRODUCTION}

Knowledge of physiologic specialization of Septoria tritici on wheat is important before planning any genetic breeding program to obtain resistant varieties and to design efficient strategies for control (Eyal et al, 1983).

This septoriosis causes highly significant yield losses (Mann et al, 1983; Eyal et al, 1987) and is often considered as a limiting factor (Ramirez and Caglevic, 1983).

Several authors (Ballantyne, 1983; Rosielle and Boyd, 1983), emphasize the need to carry out research work on physiologic specialization of $S$ tritici to clarify the existing contradictions on this subject. Isolates of the pathogen obtained in Israel behave as classic races (Eyal et al, 1983; Yechilevich-Auster et al, 1983). Physiologic specilization has also been mentioned in Australia (Ballantyne, 1983), Uruguay (Diaz, 1983) and the United States (Prestes and Hendrix, 1977).

In Argentina the 2 existing studies (Cordo and Arriaga, 1987; Perelló et al, 1987) reveal that tested isolates causes significantly different levels of infection for each isolate $x$ cultivar (CV) combination. In spite of great variability in the host-pathogen interaction, Cordo and Arriaga (1987) indicate that host response differences were not great. Due to this fact, the isolates were not classified into physiologic races. Besides, although there was a certain degree of specialization (Perelló et al, 1987), differences in behavior of $\mathrm{cvs}$ and isolates assayed were not as clear and outstanding as to establish races.

Classification in races may include the introduction of new isolates or cvs (Stakman and Harrar, 1957; Buxton, 1960); also the recognition and grouping of races depend mainly on the availability of appropriate differentials and on the correct sampling of the pathogen under study (Stakman and Harrar, 1957). Summing up, the purpose of this study is, first, to identify physiologic races of Septoria tritici with the introduction of a wheat differential set selected in Israel, and second, to analyze varietal reaction using 2 different variables (percentage of necrotic lesion with pycnidia, and pycnidial coverage) to compare their efficiency in isolate differentiation.

\section{MATERIALS AND METHODS}

\section{Isolates' origin}

This study evaluated the virulence of $10 \mathrm{~S}$ tritici isolates. They were collected in 9 localities of Buenos Aires ( 3 wheat ecological subregions: South II, IV and V). The isolates (numbered as follows: $I_{11}, l_{12}, l_{13}, l_{16}$, $I_{19}, I_{20}, I_{21}, I_{22}, I_{23}$ and $I_{25}$ ) were obtained from the following cvs and regions (fig 1 ).

\section{Differential hosts}

Seventeen wheat cvs with different resistance in the field and genealogy were used. Also, 10 international differentials selected in Israel were included to identify physiologic races of $S$ tritici as 7 commercial Argentine cvs already tested (Perelló et al, 1987) (table I).

\section{Inoculation and evaluation}

The isolates were obtained from leaves, which presented typical lesions with pycnidia, disinfected and incubated on potato-dextrose agar (PDA). Pycnidia developed spore cirrhi; from these single spore isolates were cultivated on water agar. On day 6 they were replicated in slant tubes onto modified malt agar $(30 \mathrm{~g}$ malt extract, $5 \mathrm{~g}$ mycological peptone, $2 \mathrm{~g}$ yeast extract and $1000 \mathrm{ml}$ distilled water) for sporulation. On day 9 a mucous mass of conidia developed; afterwards, they were used for inoculation. Spore suspension was adjusted to $1 \times 10^{7}$ spores $/ \mathrm{ml}$ concentration in $350 \mathrm{ml}$ distilled water. The design was a randomized block design with 4 replications; each pot $(12 \mathrm{~cm}$ diameter; $15 \mathrm{~cm}$ depth) was considered as 1 replication. Ten seeds were sown per pot; after emergence, 


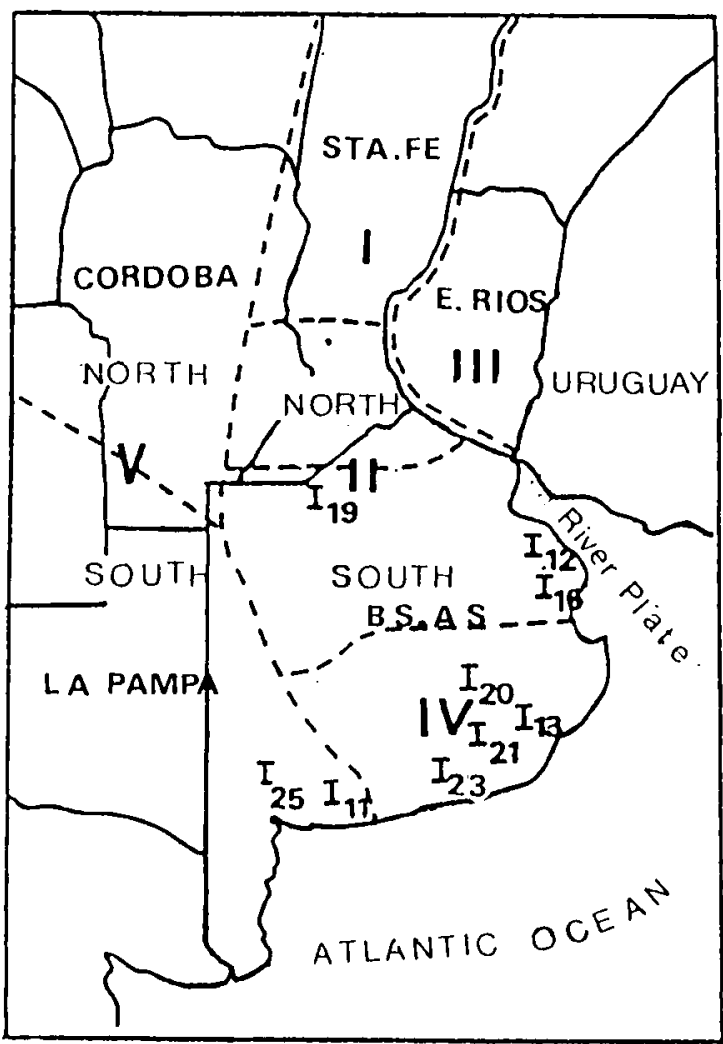

Fig 1. Map of Argentine wheat areas (I, III, IV; II and V north and south) showing $S$ tritici isolates collection sites. $l_{11}$ (Cnel Dorrego), $I_{13}$ (Miramar) and $I_{22}$ (Bavio) from Buck Napostá origin; $I_{12}$ (La Plata) from Norking $70 ; I_{16}$ (Bavio) from Buck Pucará; $l_{19}$ (Rojas) from Victoria INTA; $I_{20}$ (Tandil) from Buck Nandú; $I_{21}$ (Miramar) from Retacón INTA; $I_{23}$ (Necochea) from Buck Ombú and $\mathrm{I}_{25}$ (Bahia Blanca) from Cooperación Cabildo.
5 seedlings were selected. At the 3rd expanded leaf stage with the 4th leaf emerging ( $\approx$ day 25 ), the seedlings were sprayed with the inoculation until soaking and then taken to a humid chamber for $96 \mathrm{~h}$. The assay was carried out in a greenhouse under controlled conditions (temperature : minimum mean, $10^{\circ} \mathrm{C}$; maximum mean, $20^{\circ} \mathrm{C}$; relative humidity: $25-80$ ).

Symptoms were evaluated 28 days after inoculation time on the 3rd leaf of 20 plants for each isolate $x$ cv combination.

Varietal reaction was determined through 2 variables: pycnidial leaf coverage percentage (PC) and necrotic lesion percentage $(\mathrm{NL})$ on the leaves, both in reference to total leaf surface. Similarly, qualitative differences were observed in the type of lesion developed.

\section{Data analysis}

Friedman's test (1937) was used in the statistical analysis of data to verify 2 hypotheses: $\mathrm{H}_{0}$, every isolate acted similarly with respect to $1 \mathrm{cv}$, and $\mathrm{H}_{1}$, at least 1 isolate behaved differently with respect to $1 \mathrm{cv}$.

Friedman's test was applied for each of the $17 \mathrm{cvs}$ using PC and NL separately to determine whether the discriminating information they supplied was similar. Similarities and differences in isolate behavior within cvs were determined by Miller's (Pirie, 1974) multiple comparison algorithm. After this, the isolates that presented similar virulence were gathered by clustering "without supervision" method (Hartigan, 1975).

Table I. Wheat cultivars assayed to test $S$ tritici isolate virulence.

Code Cultivar Genealogy

\begin{tabular}{|c|c|c|}
\hline 1 & Frontana (C.I. 12470) & Fronteira x Mentana \\
\hline 2 & Klein Titán (C.I. 15615) & Barleta 7D x Americano 44D \\
\hline 3 & Lakhisch & (Yaktana/Norin 10/Brévor/3/Florence Aurore) \\
\hline 4 & Séri 82 & (Kavkaz $\times$ Buho "S") $\times$ (Kalyanzola $\times$ Bluebird) \\
\hline 5 & Veranapolis (AUS 1553) & Trintecinco x B 2017-37 \\
\hline 6 & lassul 20 & Colonias//Frontana/Kenia 58 \\
\hline 7 & Colotana $(\mathrm{Cl} 13556)$ & Colonista $\times$ Frontana \\
\hline 8 & Bobwhite "S" & $\begin{array}{l}\text { Aurora x Kalyanzola-Bluebird/Wood } \\
\text { Pecker "S" (CM 33203-K-9M-ZY-IM-IY-IM-OY) }\end{array}$ \\
\hline 9 & Nursit 163 & \\
\hline 10 & Kavkaz (KVZ)-K 4500 & $\begin{array}{l}\text { LA4 SWO 176-3M-IY-IOY-IY-IM-OY-OPts } \\
\text { (CIMMYT, } 12 \text { ISEPTON//99) }\end{array}$ \\
\hline 11 & Bordenave Puán & (Cheng $160 \times$ Klein Aniversario) \\
\hline 12 & Buck Manantial & (Rafaela MAG x Buck Quequén) \\
\hline 13 & Buck Pangaré & I (B. Relén x Rafaela MAG) x B. Pampero I x Robin \\
\hline 14 & Diamante INTA & I (Sonora 64 x Tezanos Pinto) x Nai 60 I x Nar 50 \\
\hline 15 & Marcos J INTA & (Sonora 64 x Klein Reindidor) \\
\hline 16 & Buck Napostá & $\begin{array}{l}\text { (Rafaela MAG x B. Pampero x Buck Araucano } \\
\text { x Buck Quequén x Rafaela MAG). }\end{array}$ \\
\hline 17 & Klein Toledo & $\begin{array}{l}\text { (Seleccion no registrada pariente Klein Atlas } \\
\text { x selección no registrada IBagé Oiterst K 209-45 Selección I; } \\
\text { Klein O:O Selección x Klein Lucero. }\end{array}$ \\
\hline
\end{tabular}

1-10, International differential cultivars selected in Israel; 11-17, commercial Argentine cultivars. 
The isolates were arranged by Page's test (1963) according to their degree of virulence for each $\mathrm{cv}$.

A clustering algorithm $K$-mean $(K=3$ ) (Duda and Hart, 1973) was used to group in different classes the isolate $\mathrm{x} \mathrm{cv}$ interactions with similar levels of virulence susceptibility.

A variance analysis was performed for each variable under study (PC and $\mathrm{NL}$ ) to detect significant isolate $x$ cv interactions.

\section{RESULTS}

Using both variables (PC and NL), Friedman's test rejected $\mathrm{H}_{0}$ for all the cvs $(P<0.005)$. lassul 20 and Nursit 163 rejected it with higher associated probability $(0.005<P<0.01)$. The $10 \mathrm{~S}$ tritici isolates tested stood out because of their different behavior on $17 \mathrm{cvs}$. Both variables helped discriminate which isolates acted similarly and which acted differently with respect to each cv (table II).

\section{Relation pycnidial coverage-necrotic lesion}

Table III showed ranking of pycnidial coverage and necrotic lesion for every isolate $x \mathrm{cv}$ combination. Page's test showed differences in patterns of isolate virulence on hosts as on each cv in particular. Some cvs showed few symptoms (necrosis and pycnidia), eg Veranopolis, Bobwhite "S" and lassul 20, whereas some others presented abundant necrosis and numerous pycnidia, eg Lakhish with $I_{11}, l_{12}, l_{19}$ and $I_{22}$. Lakhish developed abundant necrosis and few pycnidia with $I_{13}$ and $I_{20}$, similar to Colotana with $I_{11}$ and $\mathrm{I}_{25}$, and to Buck Manantial with $\mathrm{I}_{19}$ and $\mathrm{I}_{23}$. On the contrary, Seri 82 and Marcos Juárez INTA showed small necrotic area and abundant pycnidia with $I_{11}, l_{13}, l_{19}$ and $I_{25}$, and with $I_{12}$ and $l_{13}$ respectively. Bordenave Puán, lassul 20 and Bobwhite " $S$ " showed as much NL and PC with most of the isolates tested.

There was no coincidence in the behavior pattern induced by both variables within the remaining crs.

In general, local isolates induced higher PC than NL percentage in the national cvs, eg Buck Pangaré and Marcos Juárez INTA with $\mathrm{I}_{11}, \mathrm{I}_{12}$, $I_{13}, I_{19}, I_{20}, I_{21}, I_{22}$ and $I_{23}$; Buck Napostá with $I_{19}, I_{20}$ and $I_{22}$; Klein Toledo with $I_{11}, I_{13}, I_{16}, I_{19}$, $\mathrm{I}_{22}$ and $\mathrm{I}_{23}$.

Three isolates $\left(I_{20}, I_{21}\right.$ and $\left.I_{25}\right)$ were more virulent on Frontana than on Klein Titán, whereas $\mathrm{I}_{12}, \mathrm{I}_{16}$ and $\mathrm{I}_{23}$ were more virulent on the latter.

From all the isolates assayed, $l_{11}$ and $l_{19}$ induced a higher number of susceptible reactions, whereas $\mathrm{I}_{21}$ and $\mathrm{I}_{25}$ behaved as the least virulent isolates presenting the highest percentage of resistant reactions on the $17 \mathrm{cvs}$ assayed.

Table II. Multiple comparison matrix (Miller's test) to group isolates presenting similar behavior (identified by the same letter).

\begin{tabular}{lllllllllll}
\hline Isolate & $I_{11}$ & $I_{12}$ & $I_{13}$ & $I_{16}$ & $I_{19}$ & $I_{20}$ & $I_{21}$ & $I_{22}$ & $I_{23}$ & $I_{25}$ \\
Cultivar & & & & & & & & & & \\
& & & & & & & & & & \\
\hline & $\mathrm{Aa}$ & $\mathrm{Bb}$ & $\mathrm{Bb}$ & $\mathrm{Cb}$ & $\mathrm{Ab}$ & $\mathrm{Aa}$ & $\mathrm{Ab}$ & $\mathrm{Ba}$ & $\mathrm{Cb}$ & $\mathrm{Bb}$ \\
Frontana & $\mathrm{Aa}$ & $\mathrm{Bb}$ & $\mathrm{Cb}$ & $\mathrm{Ba}$ & $\mathrm{Ba}$ & $\mathrm{Ba}$ & $\mathrm{Ac}$ & $\mathrm{Ac}$ & $\mathrm{Ba}$ & $\mathrm{Ca}$ \\
Klein Titán & $\mathrm{Aa}$ & $\mathrm{Aa}$ & $\mathrm{Ba}$ & $\mathrm{Bb}$ & $\mathrm{Ac}$ & $\mathrm{Ba}$ & $\mathrm{Ca}$ & $\mathrm{Ac}$ & $\mathrm{Ad}$ & $\mathrm{Cb}$ \\
Lakhish & $\mathrm{Aa}$ & $\mathrm{Ab}$ & $\mathrm{Aa}$ & $\mathrm{Ba}$ & $\mathrm{Ba}$ & $\mathrm{Ab}$ & $\mathrm{Bb}$ & $\mathrm{Cb}$ & $\mathrm{Cb}$ & $\mathrm{Cb}$ \\
Seri 82 & $\mathrm{Aa}$ & $\mathrm{Ba}$ & $\mathrm{Ca}$ & $\mathrm{Ab}$ & $\mathrm{Ab}$ & $\mathrm{Ab}$ & $\mathrm{Bb}$ & $\mathrm{Bb}$ & $\mathrm{Aa}$ & $\mathrm{Bb}$ \\
Veranópolis & $\mathrm{Aa}$ & $\mathrm{Aa}$ & $\mathrm{Bb}$ & $\mathrm{Ab}$ & $\mathrm{Aa}$ & $\mathrm{Ba}$ & $\mathrm{Aa}$ & $\mathrm{Aa}$ & $\mathrm{Ba}$ & $\mathrm{Aa}$ \\
lassul 20 & $\mathrm{Aa}$ & $\mathrm{Ba}$ & $\mathrm{Ab}$ & $\mathrm{Ac}$ & $\mathrm{Ca}$ & $\mathrm{Cb}$ & $\mathrm{Ca}$ & $\mathrm{Ba}$ & $\mathrm{Ba}$ & $\mathrm{Ab}$ \\
Colotana & $\mathrm{Aa}$ & $\mathrm{Bb}$ & $\mathrm{Aa}$ & $\mathrm{Ba}$ & $\mathrm{Aa}$ & $\mathrm{Aa}$ & $\mathrm{Aa}$ & $\mathrm{Ba}$ & $\mathrm{Aa}$ & $\mathrm{Aa}$ \\
Bobwhite "S" & $\mathrm{Aa}$ & $\mathrm{Aa}$ & $\mathrm{Ba}$ & $\mathrm{Aa}$ & $\mathrm{Aa}$ & $\mathrm{Ba}$ & $\mathrm{Ba}$ & $\mathrm{Cb}$ & $\mathrm{Ba}$ & $\mathrm{Ba}$ \\
Nursit 163 & $\mathrm{Aa}$ & $\mathrm{Aa}$ & $\mathrm{Bb}$ & $\mathrm{Bb}$ & $\mathrm{Ac}$ & $\mathrm{Bb}$ & $\mathrm{Cb}$ & $\mathrm{Cb}$ & $\mathrm{Dc}$ & $\mathrm{Bc}$ \\
Kavkaz 4500 & $\mathrm{Aa}$ & $\mathrm{Ab}$ & $\mathrm{Ab}$ & $\mathrm{Ba}$ & $\mathrm{Ba}$ & $\mathrm{Ba}$ & $\mathrm{Ba}$ & $\mathrm{Ca}$ & $\mathrm{Cc}$ & $\mathrm{Cc}$ \\
Bordenave Puán & $\mathrm{Aa}$ & $\mathrm{Ab}$ & $\mathrm{Ac}$ & $\mathrm{Ba}$ & $\mathrm{Bd}$ & $\mathrm{Ba}$ & $\mathrm{Ab}$ & $\mathrm{Bd}$ & $\mathrm{Ab}$ & $\mathrm{Ba}$ \\
Buck Manantial & $\mathrm{Aa}$ & $\mathrm{Aa}$ & $\mathrm{Ba}$ & $\mathrm{Cb}$ & $\mathrm{Aa}$ & $\mathrm{Bd}$ & $\mathrm{Dc}$ & $\mathrm{Aa}$ & $\mathrm{Bd}$ & $\mathrm{Ba}$ \\
Buck Pangaré & $\mathrm{Aa}$ & $\mathrm{Bd}$ & $\mathrm{Bd}$ & $\mathrm{Bd}$ & $\mathrm{Cb}$ & $\mathrm{Ac}$ & $\mathrm{Ac}$ & $\mathrm{Ac}$ & $\mathrm{Ba}$ & $\mathrm{Cb}$ \\
Diamante INTA & $\mathrm{Aa}$ & $\mathrm{Ab}$ & $\mathrm{Ab}$ & $\mathrm{Bc}$ & $\mathrm{Aa}$ & $\mathrm{Bc}$ & $\mathrm{Bc}$ & $\mathrm{Aa}$ & $\mathrm{Cb}$ & $\mathrm{Db}$ \\
Marcos Juárez INTA & $\mathrm{Aa}$ & $\mathrm{Aa}$ & $\mathrm{Aa}$ & $\mathrm{Aa}$ & $\mathrm{Cb}$ & $\mathrm{Cb}$ & $\mathrm{Bc}$ & $\mathrm{Ac}$ & $\mathrm{Bc}$ & $\mathrm{Bc}$ \\
Buck Napostá & $\mathrm{Aa}$ & $\mathrm{Bb}$ & $\mathrm{Cb}$ & $\mathrm{Da}$ & $\mathrm{Ac}$ & $\mathrm{Ca}$ & $\mathrm{Ba}$ & $\mathrm{Ac}$ & $\mathrm{Da}$ & $\mathrm{Bb}$ \\
Klein Toledo & & & & & & & & & & \\
\hline
\end{tabular}


Table III. Virulence patterns of the $10 \mathrm{~S}$ tritici isolates on the differential set for the 2 variables analyzed.

\begin{tabular}{|c|c|c|c|c|c|c|c|c|c|c|}
\hline $\begin{array}{l}\text { Isolate } \\
\text { Cultivar }\end{array}$ & $I_{11}$ & $l_{12}$ & $I_{13}$ & $I_{16}$ & $I_{19}$ & $I_{20}$ & $I_{21}$ & $I_{22}$ & $I_{23}$ & $I_{25}$ \\
\hline Frontana & $\begin{array}{c}3 \\
(2)\end{array}$ & $\begin{array}{c}2 \\
(1)\end{array}$ & $\begin{array}{c}2 \\
(1)\end{array}$ & $\begin{array}{c}1 \\
(1)\end{array}$ & $\begin{array}{c}3 \\
(1)\end{array}$ & $\begin{array}{c}3 \\
(2)\end{array}$ & $\begin{array}{c}3 \\
(1)\end{array}$ & $\begin{array}{c}2 \\
(2)\end{array}$ & $\begin{array}{c}1 \\
(1)\end{array}$ & $\begin{array}{c}2 \\
(1)\end{array}$ \\
\hline Klein Titán & $\begin{array}{c}2 \\
(2)\end{array}$ & $\begin{array}{c}3 \\
(3)\end{array}$ & $\begin{array}{c}1 \\
\text { (3) }\end{array}$ & $\begin{array}{c}3 \\
(2)\end{array}$ & $\begin{array}{c}3 \\
(2)\end{array}$ & $\begin{array}{c}2 \\
(2)\end{array}$ & $\begin{array}{c}2 \\
(1)\end{array}$ & $\begin{array}{c}2 \\
(1)\end{array}$ & $\begin{array}{c}3 \\
(2)\end{array}$ & $\begin{array}{c}1 \\
(2)\end{array}$ \\
\hline Lakhish & $\begin{array}{l}3 \\
\text { (3) }\end{array}$ & $\begin{array}{c}3 \\
(3)\end{array}$ & $\begin{array}{c}1 \\
\text { (3) }\end{array}$ & $\begin{array}{c}1 \\
\text { (2) }\end{array}$ & $\begin{array}{c}3 \\
(4)\end{array}$ & $\begin{array}{c}1 \\
\text { (3) }\end{array}$ & $\begin{array}{c}2 \\
(3)\end{array}$ & $\begin{array}{l}3 \\
(4)\end{array}$ & $\begin{array}{c}3 \\
(1)\end{array}$ & $\begin{array}{c}2 \\
(2)\end{array}$ \\
\hline Seri 82 & $\begin{array}{c}3 \\
(1)\end{array}$ & $\begin{array}{c}3 \\
(2)\end{array}$ & $\begin{array}{c}3 \\
(1)\end{array}$ & $\begin{array}{c}1 \\
\text { (1) }\end{array}$ & $\begin{array}{c}3 \\
(1)\end{array}$ & $\begin{array}{c}1 \\
(2)\end{array}$ & $\begin{array}{c}2 \\
(2)\end{array}$ & $\begin{array}{c}2 \\
(2)\end{array}$ & $\begin{array}{c}2 \\
(2)\end{array}$ & $\begin{array}{c}4 \\
(2)\end{array}$ \\
\hline Veranópolis & $\begin{array}{c}1 \\
(1)\end{array}$ & $\begin{array}{c}2 \\
(2)\end{array}$ & $\begin{array}{l}2 \\
(2)\end{array}$ & $\begin{array}{c}1 \\
(1)\end{array}$ & $\begin{array}{c}1 \\
(1)\end{array}$ & 2 & $\begin{array}{c}2 \\
(1)\end{array}$ & 2 & 1 & $\begin{array}{l}2 \\
(1)\end{array}$ \\
\hline lassul 20 & $\begin{array}{c}1 \\
\text { (1) }\end{array}$ & $\begin{array}{c}1 \\
(1)\end{array}$ & $\begin{array}{c}2 \\
(2)\end{array}$ & $\begin{array}{l}1 \\
(2)\end{array}$ & $\begin{array}{c}1 \\
\text { (1) }\end{array}$ & $\begin{array}{c}2 \\
(1)\end{array}$ & $\begin{array}{c}1 \\
(1)\end{array}$ & $\begin{array}{l}1 \\
\text { (1) }\end{array}$ & $\begin{array}{c}2 \\
(1)\end{array}$ & $\begin{array}{c}1 \\
\text { (1) }\end{array}$ \\
\hline Colotana & $\begin{array}{c}1 \\
\text { (3) }\end{array}$ & $\begin{array}{c}2 \\
(3)\end{array}$ & $\begin{array}{c}1 \\
(2)\end{array}$ & $\begin{array}{c}1 \\
\text { (1) }\end{array}$ & $\begin{array}{c}3 \\
(3)\end{array}$ & $\begin{array}{c}3 \\
(2)\end{array}$ & $\begin{array}{c}3 \\
(3)\end{array}$ & $\begin{array}{c}2 \\
\text { (3) }\end{array}$ & $\begin{array}{c}2 \\
(3)\end{array}$ & $\begin{array}{c}1 \\
\text { (3) }\end{array}$ \\
\hline Bobwhite "S" & $\begin{array}{c}1 \\
(1)\end{array}$ & $\begin{array}{c}2 \\
(2)\end{array}$ & $\begin{array}{c}1 \\
\text { (1) }\end{array}$ & $\begin{array}{c}2 \\
\text { (1) }\end{array}$ & $\begin{array}{c}1 \\
\text { (1) }\end{array}$ & $\begin{array}{c}1 \\
(1)\end{array}$ & $\begin{array}{c}1 \\
\text { (1) }\end{array}$ & $\begin{array}{c}2 \\
(1)\end{array}$ & $\begin{array}{c}1 \\
\text { (1) }\end{array}$ & $\begin{array}{c}1 \\
\text { (1) }\end{array}$ \\
\hline Nursit 163 & $\begin{array}{c}2 \\
(2)\end{array}$ & $\begin{array}{c}2 \\
(2)\end{array}$ & $\begin{array}{c}1 \\
\text { (2) }\end{array}$ & $\begin{array}{c}2 \\
(2)\end{array}$ & $\begin{array}{c}2 \\
(2)\end{array}$ & $\begin{array}{c}1 \\
(2)\end{array}$ & $\begin{array}{c}1 \\
(2)\end{array}$ & $\begin{array}{c}3 \\
(1)\end{array}$ & $\begin{array}{c}1 \\
(2)\end{array}$ & $\begin{array}{c}2 \\
(2)\end{array}$ \\
\hline Kavkaz 4500 & $\begin{array}{c}2 \\
\text { (1) }\end{array}$ & $\begin{array}{c}2 \\
(1)\end{array}$ & $\begin{array}{c}3 \\
(2)\end{array}$ & $\begin{array}{l}3 \\
(2)\end{array}$ & $\begin{array}{c}2 \\
\text { (3) }\end{array}$ & $\begin{array}{c}3 \\
(2)\end{array}$ & $\begin{array}{c}1 \\
\text { (2) }\end{array}$ & $\begin{array}{c}1 \\
(1)\end{array}$ & $\begin{array}{c}4 \\
(2)\end{array}$ & $\begin{array}{c}3 \\
(2)\end{array}$ \\
\hline Bordenave Puán & $\begin{array}{c}3 \\
(2)\end{array}$ & $\begin{array}{c}3 \\
(3)\end{array}$ & $\begin{array}{c}3 \\
(3)\end{array}$ & $\begin{array}{c}2 \\
(2)\end{array}$ & $\begin{array}{c}2 \\
(2)\end{array}$ & $\begin{array}{c}2 \\
(2)\end{array}$ & $\begin{array}{c}2 \\
(2)\end{array}$ & $\begin{array}{c}1 \\
(2)\end{array}$ & $\begin{array}{c}1 \\
\text { (1) }\end{array}$ & $\begin{array}{c}1 \\
\text { (1) }\end{array}$ \\
\hline Buck Manantial & $\begin{array}{c}1 \\
(3)\end{array}$ & $\begin{array}{c}1 \\
\text { (2) }\end{array}$ & $\begin{array}{c}1 \\
(1)\end{array}$ & $\begin{array}{c}2 \\
\text { (3) }\end{array}$ & $\begin{array}{c}2 \\
(4)\end{array}$ & $\begin{array}{c}2 \\
(2)\end{array}$ & $\begin{array}{c}1 \\
\text { (2) }\end{array}$ & $\begin{array}{c}2 \\
(2)\end{array}$ & $\begin{array}{c}1 \\
\text { (1) }\end{array}$ & $\begin{array}{c}2 \\
\text { (1) }\end{array}$ \\
\hline Buck Pangaré & $\begin{array}{c}3 \\
(2)\end{array}$ & $\begin{array}{c}3 \\
(2)\end{array}$ & $\begin{array}{c}2 \\
(2)\end{array}$ & $\begin{array}{c}1 \\
(1)\end{array}$ & $\begin{array}{c}3 \\
(2)\end{array}$ & $\begin{array}{c}2 \\
(1)\end{array}$ & $\begin{array}{c}4 \\
(3)\end{array}$ & $\begin{array}{c}3 \\
(2)\end{array}$ & $\begin{array}{c}2 \\
(1)\end{array}$ & $\begin{array}{c}2 \\
(2)\end{array}$ \\
\hline Diamante INTA & $\begin{array}{c}1 \\
\text { (2) }\end{array}$ & $\begin{array}{c}2 \\
(3)\end{array}$ & $\begin{array}{c}2 \\
\text { (3) }\end{array}$ & $\begin{array}{c}2 \\
(3)\end{array}$ & $\begin{array}{c}3 \\
(3)\end{array}$ & $\begin{array}{c}1 \\
\text { (1) }\end{array}$ & $\begin{array}{c}1 \\
\text { (1) }\end{array}$ & $\begin{array}{c}1 \\
\text { (1) }\end{array}$ & $\begin{array}{c}2 \\
(2)\end{array}$ & $\begin{array}{c}3 \\
(3)\end{array}$ \\
\hline Marcos Juárez INTA & $\begin{array}{c}4 \\
(3)\end{array}$ & $\begin{array}{c}4 \\
(2)\end{array}$ & $\begin{array}{c}4 \\
(2)\end{array}$ & $\begin{array}{c}2 \\
(1)\end{array}$ & $\begin{array}{c}4 \\
(3)\end{array}$ & $\begin{array}{c}2 \\
(1)\end{array}$ & $\begin{array}{c}2 \\
\text { (1) }\end{array}$ & $\begin{array}{c}4 \\
(3)\end{array}$ & $\begin{array}{c}3 \\
(2)\end{array}$ & $\begin{array}{c}1 \\
\text { (1) }\end{array}$ \\
\hline Buck Napostá & $\begin{array}{c}2 \\
(3)\end{array}$ & $\begin{array}{c}2 \\
\text { (3) }\end{array}$ & $\begin{array}{c}2 \\
\text { (3) }\end{array}$ & $\begin{array}{c}2 \\
(3)\end{array}$ & $\begin{array}{c}3 \\
(2)\end{array}$ & $\begin{array}{c}3 \\
(2)\end{array}$ & $\begin{array}{c}1 \\
(1)\end{array}$ & $\begin{array}{c}2 \\
(1)\end{array}$ & $\begin{array}{c}1 \\
(1)\end{array}$ & $\begin{array}{c}1 \\
\text { (1) }\end{array}$ \\
\hline Klein Toledo & $\begin{array}{c}4 \\
(2)\end{array}$ & $\begin{array}{c}1 \\
(1)\end{array}$ & $\begin{array}{c}2 \\
(1)\end{array}$ & $\begin{array}{l}3 \\
(2)\end{array}$ & $\begin{array}{c}4 \\
(3)\end{array}$ & $\begin{array}{c}2 \\
(2)\end{array}$ & $\begin{array}{c}1 \\
(2)\end{array}$ & $\begin{array}{c}4 \\
(3)\end{array}$ & $\begin{array}{l}3 \\
(2)\end{array}$ & $\begin{array}{c}1 \\
(1)\end{array}$ \\
\hline
\end{tabular}

PC: values without parentheses; NL: values within parentheses. Indices 1-4 indicate increasing virulence induced by the isolates in each variety $(P=0.005)$.

\section{Isolate $\times$ cultivar interaction}

Isolate $x \mathrm{cv}$ interactions, with similar virulencesusceptibility levels, were grouped into 3 classes for PC (table IV) and 2 classes for NL (table V). In general, national cvs showed a degree of higher susceptibility than the international cvs.

Seri 82, lassul 20, Bobwhite "S" and Nursit 163 were found to be the most resistant cvs with respect to every isolate.

The fact than $K$-mean algorithm separated data into more classes for PC suggested that it supplied more discriminatory information.
The analysis of variance for PC indicated significantly different behavior in isolates and cvs and, also significant interaction between them $(P=0.01)$ (table VI). For NL, it indicated that only the source of variance "cultivar" was significant $(P=0.01)$ (table VII).

\section{DISCUSSION}

Van der Plank (1968) introduced 2 methods to determine differential interactions. The first one referred to variance analysis to test significance of isolate $x \mathrm{cv}$ interaction. The second referred 
Table IV. Clustering analysis for PC variable.

\begin{tabular}{|c|c|c|c|c|c|c|c|c|c|c|}
\hline $\begin{array}{l}\text { Isolate } \\
\text { Cultivar }\end{array}$ & $I_{11}$ & $I_{12}$ & $I_{13}$ & $I_{16}$ & $I_{19}$ & $I_{20}$ & $I_{21}$ & $I_{22}$ & $I_{23}$ & $l_{25}$ \\
\hline Frontana & $\star \star \star \star$ & $\star \star * *$ & $\star *$ & $* * *$ & $* * *$ & $\star \star \star \star$ & $\star \star \star \star$ & $* * *$ & $* *$ & $\star \star$ \\
\hline Klein Titán & $\star \star *$ & $\star \star \star \star$ & * & $\star \star \star$ & $\star \star \star \star$ & $\star \star$ & $\star \star \star$ & $\star \star \star *$ & $* \star \star$ & * \\
\hline Lakhish & $\star \star \star \star$ & 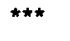 & $\star \star *$ & $\star \star$ & $\star \star \star \star$ & $\star \star$ & $\star \star \star$ & $* * *$ & $\star \star \star \star *$ & $\star \star \star \star$ \\
\hline Seri 82 & $\star \star$ & $\star \star *$ & $\star \star \star *$ & $\star$ & $\star *$ & $\star$ & $*$ & * & $\star \star \star$ & $\star \star$ \\
\hline Veranópolis & $*$ & $* *$ & $* * *$ & * & * & $\star *$ & $\star \star *$ & $* *$ & ** & $\star \star \star \star$ \\
\hline lassul 20 & $*$ & $*$ & $\star$ & * & $*$ & $\star \star$ & $\star$ & $\star$ & $* \star$ & $\star$ \\
\hline Colotana & $*$ & * & * & $*$ & $* * *$ & $\star \star$ & $\star \star$ & $*$ & * & $*$ \\
\hline Bobwhite "S" & $*$ & $\star \star$ & * & ** & $*$ & * & $\star$ & $\star \star$ & * & $*$ \\
\hline Nursit 163 & * & $\star \star$ & $\star$ & ** & $\star \star$ & $\star$ & $\star$ & $\star \star$ & $\star *$ & $\star \star \star \star$ \\
\hline Kavkaz 4500 & * & $*$ & $\star *$ & $\star \star$ & * & $\star \star$ & $\star \star$ & * & $\star \star \star \star$ & $\star \star \star$ \\
\hline Bordenave Puán & $* * \star$ & 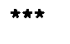 & $\star \star \star \star$ & ** & 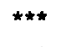 & $\star \star *$ & ** & * & * & * \\
\hline Buck Manantial & $\star \star$ & $\star \star$ & $\star \star$ & $\star \star \star$ & $\star \star \star$ & $\star \star \star$ & $\star \star \star$ & $\star \star \star$ & ** & $\star \star \star \star$ \\
\hline Buck Pangaré & $\star \star \star$ & $* * *$ & $* * *$ & $\star *$ & 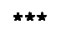 & $\star * *$ & $\star \star \star *$ & $\star \star \star$ & 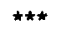 & $\star \star \star *$ \\
\hline Diamante INTA & $\star \star \star$ & $\star \star \star *$ & $\star * \star *$ & $* * *$ & $* * *$ & $\star \star \star *$ & $\star \star * *$ & $* * *$ & $* * *$ & $\star \star \star \star$ \\
\hline Marcos Juárez INTA & 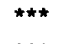 & $\star \star \star *$ & $* \star \star$ & $* * *$ & $\star \star \star \star$ & $\star \star$ & $\star \star \star$ & $\star * *$ & $\star \star \star \star$ & $\star \star$ \\
\hline Buck Napostá & $\star \star \star \star$ & $\star \star \star$ & $\star \star \star \star$ & $\star \star$ & $\star \star \star \star$ & $\star \star \star \star$ & $\star \star$ & $* * *$ & ** & 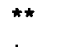 \\
\hline Klein Toledo & 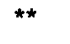 & $*$ & $\star$ & $\star *$ & $* * *$ & $* *$ & * & $\star *$ & $\star *$ & * \\
\hline
\end{tabular}

Mean cluster $1\left(^{*}\right): 5.246163 ;$ mean Cluster $2\left(^{* *}\right): 12.5345 ;$ mean cluster $3\left({ }^{* * *}\right): 28.1532$.

to classifying pathogen isolates according to their virulence on the assayed hosts. Significant statistical changes in isolates "ranking" on different cvs showed differential interaction.
In this work significant $P C$ value indicated differential interaction which might be considered as evidence of specialization; however, NL presented no significant differential interaction for

Table V. Clustering analysis for NL variable.

$\begin{array}{llllllllllllllll} & \text { Isolate } & l_{11} & l_{12} & l_{13} & l_{16} & l_{19} & l_{20} & l_{21} & l_{22} & l_{23} & I_{25}\end{array}$

Cultivar

\begin{tabular}{|c|c|c|c|c|c|c|c|c|c|}
\hline Frontana & $\star \star \star$ & $\star \star \star$ & $\star \star \star *$ & $\star \star \star$ & $\star \star \star$ & $\star \star \star$ & $\star \star \star$ & $\star \star \star$ & $* * *$ \\
\hline Klein Titán & $* * *$ & $\star \star \star *$ & $\star \star \star *$ & 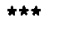 & 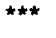 & $\star \star * *$ & $* * *$ & $\star \star \star$ & $\star \star \star *$ \\
\hline Lakhish & $\star \star \star *$ & $\star \star *$ & $\star \star *$ & $\star \star \star *$ & $* * *$ & $\star * *$ & $\# * \star$ & $\star \star *$ & $* *$ \\
\hline Seri 82 & $\star \star$ & $\star \star \star$ & $\star \star$ & $\star \star$ & $\star \star$ & $* \star *$ & $* * *$ & $\star \star \star$ & $\star \star \star$ \\
\hline Veranópolis & $\star \star \star *$ & $\star \star \star$ & $\star \star \star$ & $\star \star \star$ & $\star \star \star$ & ** & $\star * \star$ & $\star \star$ & $\star \star \star$ \\
\hline lassul 20 & $\star \star \star *$ & $\star * *$ & $* * *$ & $\star * *$ & $\star \star \star$ & $\star \star \star$ & $\star \star \star *$ & $\star * *$ & $\star \star \star$ \\
\hline Colotana & $\star * *$ & ** & 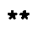 & $\star \star \star *$ & ** & 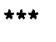 & $\star * *$ & $* * *$ & $\star \star \star$ \\
\hline Bobwhite "S" & $\star \star \star$ & $\star \star \star$ & $\star \star \star$ & $\star \star \star$ & $\star \star$ & $\star \star \star$ & $\star \star$ & $\star \star$ & $\star *$ \\
\hline Nursit 163 & $\star \star \star$ & $\star \star \star$ & $\star \star \star$ & $\star \star \star$ & $\star \star \star$ & $\star \star \star$ & $\star \star \star \star$ & $\star$ & $\star \star \star$ \\
\hline Kavkaz 4500 & $\star \star \star$ & $* * *$ & $\star \star *$ & $* * *$ & $\star \star \star *$ & 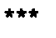 & $\star \star \star$ & $\star \star \star$ & $\star \star \star *$ \\
\hline Bordenave Puán & $\star \star \star$ & $\star \star *$ & $\star \star \star$ & $\star \star * *$ & $\star \star *$ & *** & $\star \star \star *$ & $\star \star \star$ & $\star \star *$ \\
\hline Buck Manantial & $\star \star \star$ & $\star \star \star$ & $\star \star \star$ & $\star \star \star$ & $\star \star \star$ & $\star \star \star$ & $\star \star \star$ & $\star \star \star$ & $\star \star \star *$ \\
\hline Buck Pangaré & 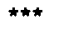 & $\star \star \star$ & $\star \star \star$ & $\star \star \star$ & $* \star *$ & $\star * *$ & $\star \star \star *$ & $* * \star$ & $* \star \star$ \\
\hline Diamante INTA & $\star * \star$ & $* * *$ & $\star * *$ & $* * *$ & $\star \star \star *$ & $* * *$ & $* * *$ & $\star \star \star$ & $\star \star \star *$ \\
\hline Marcos Juárez INTA & $\star \star \star *$ & $\star \star \star$ & $\star \star \star$ & $\star \star \star$ & $\star \star \star$ & $\star \star$ & $\star \star \star *$ & 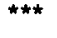 & $* * *$ \\
\hline Buck Napostá & $\star \star \star$ & $\star \star \star$ & $\star \star \star$ & $\star \star \star$ & $\star \star \star$ & $\star \star \star$ & $\star \star$ & $\star \star$ & $\star *$ \\
\hline Klein Toledo & $* *$ & $\star \star$ & $\star \star$ & $\hbar * \star$ & $\star \star \star *$ & \#* & $\star *$ & $\hbar \star \star$ & $\star \star$ \\
\hline
\end{tabular}


Table VI. Variance analysis for the variety sources under study.

\begin{tabular}{lccccc}
\hline $\begin{array}{l}\text { Source } \\
\text { of var }\end{array}$ & $\begin{array}{l}\text { Sum of } \\
\text { squares }\end{array}$ & $\begin{array}{l}\text { Degrees of } \\
\text { freedom }\end{array}$ & $\begin{array}{l}\text { Mean square } \\
\text { deviation }\end{array}$ & $\begin{array}{l}\text { Test } \\
\text { statistic }\end{array}$ & $\begin{array}{l}F \\
(0.01)\end{array}$ \\
\hline Varieties & 88084.56 & 16 & 5505.285 & 44.8806 & 1.99 \\
Isolates & 4069.327 & 9 & 452.1475 & 3.6860 & 2.41 \\
Interaction & 35224.85 & 144 & 244.617 & 1.9941 & 1.31 \\
Residuals & 396208.4 & 3230 & 122.665 & & \\
Total & 523587.2 & 3399 & & & \\
\hline
\end{tabular}

Variable : CP.

Table VII. Variance analysis for the variety sources under study.

\begin{tabular}{lccccc}
\hline $\begin{array}{l}\text { Source } \\
\text { of var }\end{array}$ & $\begin{array}{l}\text { Sum of } \\
\text { squares }\end{array}$ & $\begin{array}{l}\text { Degrees of } \\
\text { freedom }\end{array}$ & $\begin{array}{l}\text { Mean square } \\
\text { deviation }\end{array}$ & $\begin{array}{l}\text { Test } \\
\text { statistic }\end{array}$ & $\begin{array}{l}F \\
(0.01)\end{array}$ \\
\hline Varieties & 57076.32 & 16 & 3567.27 & 7.935202 & 1.99 \\
Isolates & 6805.777 & 9 & 756.1975 & 1.682121 & 2.41 \\
Interaction & 38186.86 & 144 & 265.1865 & 0.589893 & 1.31 \\
Residuals & 1452047 & 3230 & 449.5501 & & \\
Total & 1554116 & 3399 & & & \\
\hline
\end{tabular}

Variable : NL.

isolates $x$ cvs. This suggested those differences would be due to varying degrees of aggressiveness within the isolates only (Van der Plank, 1968).

Besides, the low specificity degree found within interactions as the low pathogenicity variation of isolates on some of the cvs made it difficult to classify isolates into well defined races.

Other studies revealing similar difficulties placed non classifying isolates into physiological races (Narvaez, 1957; Morales, 1958; Arjona et al, 1976; Scharen et al, 1985).

Shipton et al (1971) referred to "specialized forms" of $S$ tritici though they could not identify biotypes. Jacob' results (in Eyal et al, 1973), like those presented here, indicated that some isolates of $S$ tritici induced similar patterns of disease severity on each infected $\mathrm{cv}$; therefore, they could not show "true physiologic specialization". As was already suggested for another pathogen (Ou and Ayad, 1968), it would probably be better to describe virulence spectrum of a local isolate population than to attempt definition of different physiological races.
On relating our previous results (Cordo and Arriaga, 1987; Perelló et al, 1987) to those presented here, some strains, acting upon the same differentials, repeated patterns of behavior. Two isolates, $I_{19}$ (from Victoria INTA) and $I_{21}$ (from Retacón INTA), remained the least virulent and the most virulent, respectively. As regards cvs, in Klein Toledo (resistant to all the isolates), and Marcos Juarez INTA (susceptible) these qualities prevailed. Lack of coincidence in the other responses could be attributed, for example, to different genetics of the isolates.

Of the 2 variables analyzed, NL became a good indicator of disease development in the cvs, though in many cases the presence of aberrant lesions (with very few or no pycnidia) made it difficult to arrive at a correct interpretation. Besides, it provided less behavior differentiation among isolates than PC. The latter was a more sensitive indicator of differences within strains; it was therefore, more advisable to use it.

There have been several studies on physiologic specialization through different methodolo- 
gies (Eyal et al, 1973; Arjona et al, 1976; Díaz, 1983; Perelló et al, 1987) (differential set, age of plants, inoculum concentration, evaluation of varietal response). As a consequence, scientific information could not be compared on an international basis. All this has suggested the need to standardize working conditions so as to obtain comparable results on the interactions $S$ triticiTriticum sp.

\section{CONCLUSIONS}

The use of international varieties supplied neither higher variation range in lesion type and percentage nor more consistent varietal reactions that were sufficiently defined as to differentiate races.

The standardization of inoculating conditions and disease evaluation is necessary to assess the reproducibility of results and to prevent different interpretations of varietal reactions which might result from using different evaluation parameters.

\section{ACKNOWLEDGMENTS}

We thank $Z$ Eyal for the suggestions regarding the present work, A Pascar for advice on statistical analysis and to $C$ Burgos for technical assistance in translating this paper from Spanish into English.

\section{REFERENCES}

Arjona CO, Fuentes S, Baver LI (1976) Estudio sobre Septoria tritici Rob ex Desm, agente causal de la mancha de la hoja del trigo en México y Guatemala. In: Mem VII Congr Nac Fitopatol, Soc Mex Fitopatol AC, 8-10 March 1976, pp 27

Ballantyne B (1983) Resistance to speckled leaf blotch of wheat in southern New South Wales. Septoria of cereals. In: Proc 1983 Workshop, Montana State Univ, Bozeman, Montana, US Dept, Agric, Agric Res Serv ARS-12, April 1985, 31-32

Buxton EW (1960) Heterocariosis, saltation and adaptation. Plant Pathol 2 (10), 359-405

Cordo CA, Arriaga HO (1987) Diferencas em patogenicidade de isolados de Mycosphaerella graminicola na Argentina. Summa Phytopathol 15 (2), 121 127
Díaz MA (1983) Variabilidad patogénica de Septoria tritici Rob. ex Desm. Invest Agronom 4, 46-50

Duda RO, Hart PE (1973) Physiological specialization of Septoria tritici. Phytopathology 63, 1087-1091

Eyal Z, Amiri Z, Wahl I (1973) Physiological specialization of Septoria tritici. Phytopathology 63, 10871091

Eyal Z, Scharen AL, Prescott MJ (1983) Global "finger-printing" of Leptosphaeria nodorum (Septoria nodorum) and Mycosphaerella graminicola (Septoria tritici) pathogenicity patterns. In: Proc 1983 Workshop, Montana State Univ, Bozeman, Montana, US Dept Agric, Agric Res Serv ARS-12, April 1985, 7475

Eyal Z, Scharen AL, Prescott JM, Van Ginkel M (1987) Enfermedades del Trigo Causadas por Septoria: Conceptos y Métodos Relacionados con el Manejo de Estas Enfermedades. CIMMYT, Mexico DF, Mexico, pp 52

Friedman $M$ (1937) The use of ranks to avoid the assumption of normality implicit in the analysis of variance. J Am Stat Assoc 32, 675-701

Hartigan JA (1975) Clustering Algorithms. John Wiley and Sons, NY, pp 427

Mann CE, Rajaram S, Villareal RL (1983) Progress in breeding for Septoria tritici resistance in semidwarf spring wheat at CIMMYT. Proc US Dept Agric, Agric Res Serv ARS-12, April 1985

Morales IN (1958) Studies on Septoria leaf blotch of wheat. Diss Abstr 18, 357-358

Narvaez I (1957) Studies of Septoria leaf blotch of wheat. Doctoral Thesis, Purdue University, W Lafayette, pp 101

Ou, Ayad (1968) Pathogenic races of Pyricularia oryzae originating from single lesions and monoconidial cultures. Phytopathology 58, 179-182

Page EB (1963) Ordered hypotheses for multiple treatments: a significance test for linear ranks. $J A m$ Stat Assoc 58, 216-230

Perelló A, Babinec F, Cordo CA (1987) Especialización fisiológica en cepas argentinas de Mycosphaerella graminicola (Fuckel) Schroeter (anamorfo Septoria tritici). Taller de Septoriosis del trigo. MAP/ IPO/CIMMIT, Nov 9-14, 1987, La Estanzuela, Uruguay

Pirie WR (1974) Comparing ranking test for ordered alternatives in randomized blocks. Ann Stat 2, 374382

Prestes AM, Hendrix WJ (1977) Septoria tritici Rob ex Desm. Relaçao patogeno-hospedeiro, resposta varietal e influencia no sistema radicular do tripo. Cienc Cult (Sao Paulo) (suppl) 29, 23

Ramírez 1, Caglevic M (1983) Septoria leaf blotch of wheat in Chile. In: Proc 1983 Worshop, Montana 
Stat Univ, Bozeman, Montana. US Dept Agric, Agric Res Serv ARS-12, April 1985, 9-11

Rosielle AA, Boyd AA (1983) Genetics of hostpathogen interactions to the Septoria species of wheat. In: Proc 1984 Workshop, Montana State Univ, Bozeman, Montana. US Dept Agric, Agric Res Serv ARS-12, April 1985, 9-11

Scharen AL, Eyal Z, Huffman MD, Prescott JM (1985) The distribution and frequency of virulence genes in geographically separated population of Lepthosphaeria nodorum. Phytopathology 75, 1463-1468
Shipton WA, Boyd WR, Rosielle AA, Schearer BI (1971) The common Septoria diseases of wheat. Bot Rev 37, 231-262

Stakman EC, Harrar JG (1957) Principles of Plant Pathology. Ronald, NY, pp 581

Van der Plank JE (1968) Disease Resistance in Plants. Academic Press, NY, pp 206

Yechilevich-Auster M, Levi E, Eyal Z (1983) Assessment of interactions between cultivated and wild wheats and Septoria tritici. Phytopathology 73, 1077-1083 